\title{
FECAL CALPROTECTIN AND CRP AS BIOCHEMICAL MARKERS IN PREDICTING INFLAMMATOF BOWEL DISEASE ACTIVITY IN PATIENTS WITH ULCERATIVE COLITIS
}

\author{
Rehab Ahmed ${ }^{(1)}$, Kamal A.El-Atreb i ${ }^{(1)}$, Azza Hassan ${ }^{(2)}$, Tamer Haydara ${ }^{(3)}$, Yousry Abo-Amer ${ }^{(4)}$, \\ Mohamed El Abgeegy ${ }^{(5)}$, Mahmoud Elkadeem ${ }^{(6)}$, Sherief Abd-Elsalam ${ }^{(16)}$. \\ 1. General Medicine and Hepatology Department, National Hepatology and Tropical Medicine Research Institute (NHTMRI), \\ Cairo, Egypt. \\ 2. Medical Parasitology \& Immunology Department, National Hepatology and Tropical Medicine Research Institute, Cairo, Egypt. \\ 3. Internal Medicine Department, Faculty of Medicine, Kafrelsheikh University, Kafrelsheikh, Egypt. \\ 4. Hepatology, Gastroenterology and Infectious Diseases Department, Mahala Hepatology Teaching Hospital, Gharbia, Egypt. \\ 5. Tropical Medicine Department, National Hepatology and Tropical Medicine Research Institute, Cairo, Egypt. \\ 6. Tropical Medicine Department, Tanta University, Tanta, Egypt.
}

\section{ABSTRACT:}

Background \& Aims: The routinely used clinical activity indices alongside systemic markers of inflammation as white blood count (WBC) and erythrocyte sedimentation rate (ESR), have low sensitivity and specificity in the discrimination between inflammatory bowel disease (IBD) and irritable bowel syndrome (IBS). These limitations emphasize the need for a sensitive and specific non-invasive marker of bowel inflammation. The aim of the study was to evaluate fecal calprotectin (FC) and $\mathrm{C}$-reactive protein (CRP) as biochemical markers in predicting IBD activity in patients with ulcerative colitis (UC).

Patients $\boldsymbol{\&}$ Methods: The study included 50 patients with UC aged ranged from 12-74 years. All patients were subjected to full colonoscopy. Ileum was visualized. Each of 5 colonic segments was assessed using endoscopic component of the Mayo score. Colonic biopsies were taken from the suspected lesions for histopathological studies. Blood and stool samples were performed for both CRP and calprotectin respectively.

Results: Patients with mild activity had mean age 43.2, mean calprotectin 207.46, and mean C-reactive protein was 11.37 . While in moderate to severe group, the mean age was 42.5 , mean calprotectin 729.85 and mean CRP was 29.38. Fecal calprotectin (FC) cut off 47.5 , sensitivity $84.6 \%$, and specificity $54 \%$ and C-reactive protein cut off 7 , sensitivity $84 \%$, and specificity of 50\% in prediction of moderate to severe activity of IBD. Conclusions: Serial measurements of FC and CRP could be recommended for patients with UC to assess disease activity, induction and maintenance of remission, response to treatment, and recurrence of activity.

Keywords: Inflammatory bowel disease (IBD); Irritable bowel syndrome (IBS); Fecal calprotectin (FC); C-reactive protein (CRP); Activity.

\section{INTRODUCTION}

Ulcerative colitis (UC) is a chronic infalammatory disorder affecting colorectal mucosa. Diagnosis is usually based on combination of clinical features, laboratory tests, radiology, endoscopy, and histopathology. Evaluation of severity is still a challenge. Laboratory biomarkers are noninvasive, objective, and cheaper than other techniques. They also carry no psychological or financial burden on the patients. ${ }^{[1]}$ Differentiation between inflammatory bowel disease (IBD) and functional gut disorder remain the cornerstones of disease diagnosis and management ${ }^{[2]}$.

Different clinical activity indices in addition to systemic markers of inflammation; C-reactive protein (CRP), full blood count (FBC), platelet count, white blood count (WBC) and erythrocyte sedimentation rate (ESR), lack sensitivity and specificity in differentiating between IBD and IBS. ${ }^{[3-5]}$ Colonoscopy is the gold standard for diagnosis and assessment of IBD. However, it is costly, invasive, time consuming and carries a degree of procedural risk. These systemic biomarkers may be non-specific and can lead to a high number of unnecessary endoscopic procedures ${ }^{[4-6]}$ Additionally the use of clinical indices is mainly dependent on subjectivity and is affected by an over-reliance on patients' symptoms. $[7,8]$

C-reactive protein (CRP) is considered as one of the most important proteins expressed in acute inflammation. ${ }^{[9]}$ It is secreted by hepatocytes and maintained in low level in the circulation in healthy individuals $(<1 \mathrm{mg} / \mathrm{L})$. However, it sharply increases even reaching up to $350-400 \mathrm{mg} / \mathrm{L}$ when acute inflammation is induced by interleukin-6 (IL-6), 
tumor necrosis factor- $\alpha$ (TNF- $\alpha$ ), and IL-1 $\beta$. The level $10-40 \mathrm{mg} / \mathrm{L}$ indicates chronic inflammation or virus infection. Half-life of CRP is quite short $(19 \mathrm{~h})$; it increases rapidly and decreases sharply in acute inflammation. The situation is different in ulcerative colitis (UC) and crohn's disease (CD); CD is significantly correlated with CRP but UC is not ${ }^{[10,11]}$

Faecal inflammatory markers are extremely sensitive to gut inflammation and specific to its source. Thus, unnecessary costly endoscopes will be reduced. ${ }^{[6]}$ These are proteins secreted by granulocytes into the gut lumen during IBD. Of these calprotectin, lactoferrin, matrix metalloproteinases, and S100A12 can be measured in faeces. ${ }^{[12] .}$

Faecal calprotectin is currently the most widely used in the UK with the largest evidence-base. Faecal calprotectin (FC) is the main cytosolic protein secreted by migrating neutrophils in the inflammed bowel with high stability in faeces. It can be considered as a surrogate marker for inflammatory activity. ${ }^{[13]}$ It correlates with endoscopic activity of colonic inflammation ${ }^{[2]}$, and is useful in differential diagnostics between the disease activity and other noninflammatory conditions ${ }^{[14-15]}$

Previous studies stated that clinical score of UC patients 4 weeks after treatment significantly correlated with fecal calprotectin which also significantly correlated with the clinical score (Harvey-Bradshaw index) in CD patients 8 weeks after treatment. ${ }^{[16]}$ The aim of the study was to evaluate Fecal calprotectin and CRP as biochemical markers in predicting IBD activity in patients with ulcerative colitis.

\section{PATIENTS AND METHODS}

The study was carried out in compliance with the Declaration of Helsinki and approved by the Ethical Committee of the National Hepatology and Tropical Medicine Research Institute. It was a prospective study in which 50 patients were enrolled between July 2016 and February 2017.

All patients were subjected to colonoscopy with ileal visualization, Mayo score assessment, Mayo endoscopic subscore (MES) assessment for each of 5 colonic segments (cecum to ascending colon, transverse, descending, sigmoid colon, and rectum)., biopsies from the suspected lesions and histopathological studies, and blood and stool samples were taken for both CRP and calprotectin respectively. Bowel preparation was made using polyethylene glycol-based electrolyte solution prior to the ileocolonoscopy.

Specimens were fixed in $10 \%$ buffered formalin in room temperature and then embedded in paraffin blocks.
Sections were stained by hematoxylin and eosin. Samples were assessed for mucosal ulceration and its extent, inflammation degree and type, presence of crypt abscesses, granulomas, fibrosis, glandular distortion, goblet cells preservation, and if dysplasia was present and its degree ${ }^{(17)}$

A stool sample was taken for measurement of calprotectin. Stool samples were collected before bowel cleaning for ileocolonoscopy. All samples were stored at 20C, thawed. Calprotectin was detected using an ELISA test (Calprotectin Bühlmann ELISA; Bühlmann, Schonenbuch, Switzerland).

\section{STATISTICAL ANALYSIS}

SPSS (Statistical Package for the Social Science; IBM Corp, Armonk, NY, USA) release 22 for Microsoft Windows was used for statistical analysis. Mean and standard deviation were calculated. Mann Whitney U test was used for comparison of quantative samples non normally distributed. Receiver operating characteristic ROC curve was plotted and cut off values, sensitivities, and specificities were calculated. $\mathrm{P}$ values less than 0.05 was considered statistically significant

\section{RESULTS}

The study included 50 patients with UC aged ranged from 12-74 years. In the current study, the mean age of patients with mild activity was 43.2 , mean calprotectin (CPN) was 207.46 and mean C-reactive protein (CRP) was 11.37. While in moderate to severe group, the mean age was 42.5, mean CPN 729.85 and mean CRP was 29.38 (Figure1).

There was no statistically significant difference between patients with mild activity and ones with moderate or severe activity as regards age ( $p$ value $=0.087)$. However, significant statistical difference was detected regarding CPN ( $\mathrm{p}=0.001$ ) (mean CPN was (207.46) in mild cases and mean CPN was (729.85) in mod-severe group), and CRP ( $\mathrm{p}=0.000)$ (mean CRP in mild group was (11.37) and in mod-severe group was (29.38)). As a result, both CPN and CRP were significantly higher in moderate-severe group in relation to the group of mild activity (Table (1)). Different cut off values of calprotectin (CPN) were detected to predict moderate to severe activity (Cut off (47.5) with sensitivity $84.6 \%$, and specificity $54 \%$, cut off (85) with sensitivity $80 \%$, and specificity $62.5 \%$, cut off 191 with sensitivity $76 \%$, and specificity $70.8 \%$, cut off 219 with sensitivity $73 \%$, and specificity $79 \%$, and Cut off 429 with sensitivity $57 \%$, and specificity $87.5 \%$ ). In general, both were of high nearly equal sensitivity (CPN $84.6 \%$ vs CRP $84 \%$ ). CPN had slightly higher sensitivity and slightly better specificity $(54 \%)$ in relation to CRP (50\%) (Figure 2, 3). 
Table 1: Calprotectin and C-reactive protein values in studied patients:

\begin{tabular}{lllcc}
\hline Activity & & Age & Calprotectin & CRP \\
\hline Mild & N & 24 & 24 & 24 \\
& Mean & 43.21 & 207.46 & 11.37 \\
& Std. Deviation & 13.273 & 384.834 & 17.306 \\
\hline Mod/severe & Mean & 36.35 & 729.85 & 29.38 \\
& N & 26 & 26 & 26 \\
& Std. Deviation & 14.026 & 675.935 & 30.075 \\
\hline P value & & 0.087 & 0.001 & 0.000 \\
\hline
\end{tabular}

Figure (1): Calprotectin sensitivity and specificity in studied patients.

Figure (2): C-reactive protein sensitivity and specificity in studied patients.

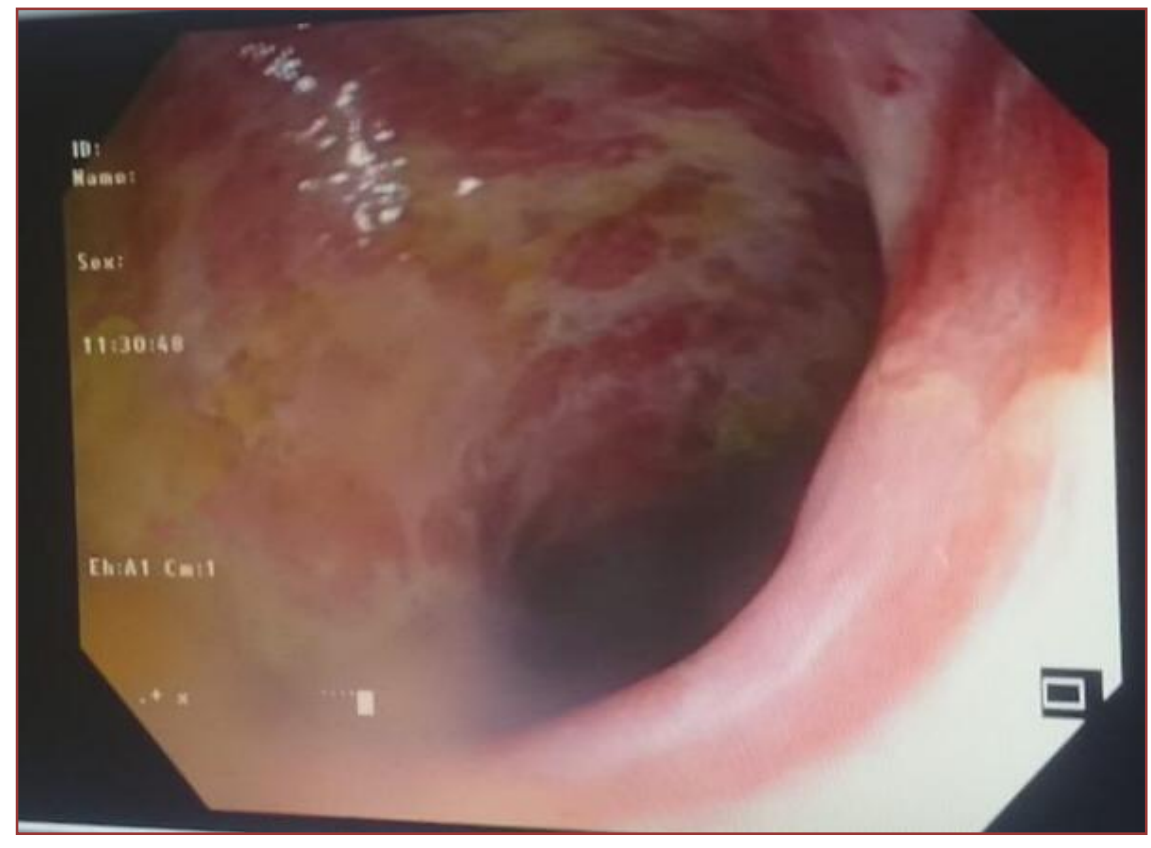

Fig. (1): Endoscopic image of ulcerative colitis.

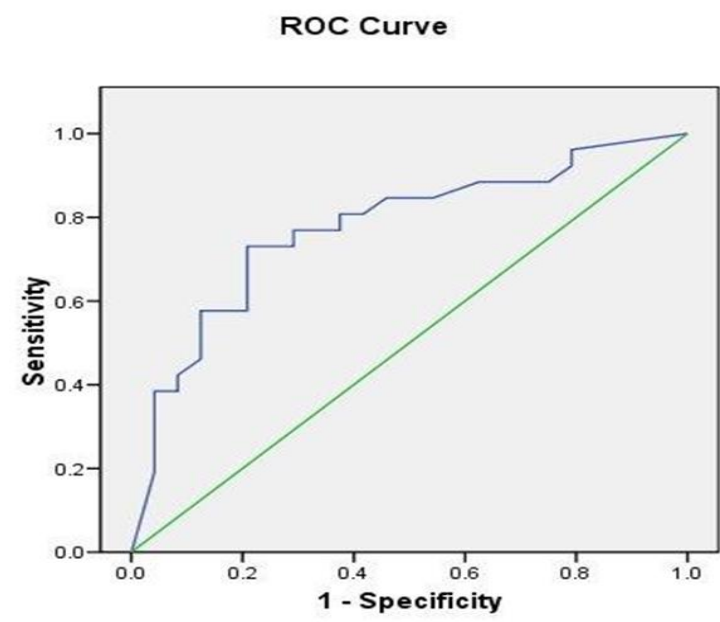

Diagonal segments are produced by ties.

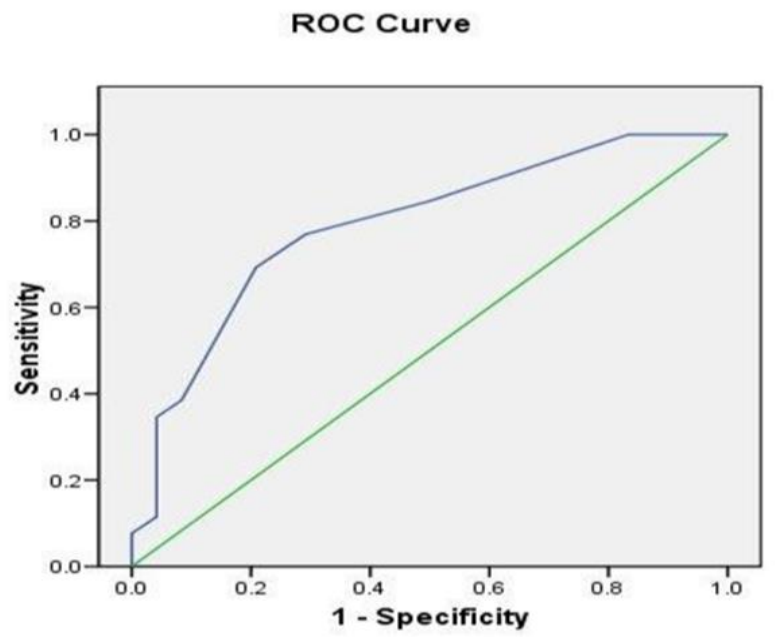

Diagonal segments are produced by ties.

Fig. (2): Calprotectin sensitivity and specificity in studied patients.

Fig. (3): C-reactive protein sensitivity and specificity in studied patients.

FECAL CALPROTECTIN AND CRP AS BIOCHEMICAL MARKERS IN PREDICTING INFLAMMATORY BOWEL DISEASE ACTIVITY IN PATIENTS WITH ULCERATIVE COLITIS 


\section{Discussion}

Production of acute-phase reactant proteins occur in the active gut inflammation including IBD; So that, these proteins may be detected in serum or stool. After regression, their concentrations will revert to normal levels but not all at the same speed. CRP has a short halflife $(19 \mathrm{~h})$ in comparison to other acute phase proteins. Therefore, it shows rapid increase and decrease rapidly after the onset and resolution of inflammation respectively ${ }^{[1]}$.

In the current study, both fecal CPN and CRP were significantly higher in moderate-severe group. Both had high sensitivity but CPN was associated with slightly higher sensitivity $(84.6 \%)$ and slightly better specificity $(54 \%)$ in relation to CRP $(50 \%)$.

Elevated ESR and CRP values in patients with active IBD have been reported by several ${ }^{[5,18]}$ Moreover, Canani et al. ${ }^{[19]}$ and Langhorst et al. ${ }^{[14]}$ reported high specificity for both CRP and ESR although they were associated with a markedly lower sensitivity than fecal markers in the diagnosis of disease activity in IBD.

Notably, fecal CPN had been shown to be a reliable marker for assessment of disease activity in IBD patients. In a study of patients with CD who underwent 140 ileocolonoscopies, fecal CPN correlated significantly with the simple endoscopic score of CD (Spearman rank correlation coefficient $\mathrm{r}=0.75)$. It was superior to $\mathrm{C}$ reactive protein, blood leukocyte levels, and $\mathrm{CD}$ activity index. Additionally, fecal CPN discriminated between the various subgroups of endoscopic activity index (inactive, mild, moderate and high activity) with sensitivity and specificity predicting endoscopic active disease (simple endoscopic score for $\mathrm{CD} \geq 4$ ) $89 \%$ and $72 \%$ respectively, at a cut-off value $70 \mu \mathrm{g} / \mathrm{g}{ }^{[20]}$.

Similarly, in a study on 134 patients with UC, fecal CPN showed a significant correlation with the Rachmilewitz endoscopic activity index ( $r=0.834)$ which was superior to the Rachmilewitz clinical activity index, C-reactive protein, and blood leukocyte levels for the detection of disease activity. Also, it was able to discriminate between various groups of endoscopic activity indices with sensitivity and specificity predicting endoscopic active disease (Rachmilewitz endoscopic activity index $\geq 4$ ) 93\%, and $71 \%$, respectively, at a cut-off $50 \mu \mathrm{g} / \mathrm{g}$ [21].

Røseth et al. showed that fecal calprotectin level correlated with endoscopic mucosal healing ${ }^{[22]}$. A metaanalysis focusing on fecal calprotectin in IBD relapse showed that the sensitivity and specificity predicting the relapse were $78 \%$ and $73 \%$, separately ${ }^{[23]}$

In agreement with study done by Mohamed et al. ${ }^{[2]}$, there was a highly significant increase in the mean value of FC in active UC patients in comparison to inactive ones. Also, there was a highly significant increase in the mean value of FC in the inactive UC patients in comparison to controls. At the cut-off value $131 \mu \mathrm{g} / \mathrm{g}, \mathrm{FC}$ had $100 \%$ accuracy, sensitivity, specificity, PPV, and NPV in differentiating UC patients from other patients with lower GI symptoms. At the cut-off value $253 \mu \mathrm{g} / \mathrm{g}$, FC had $95 \%$ accuracy, sensitivity, specificity, PPV, and NPV in differentiating active from inactive UC patients. Also in the same study, it was found that there was a highly significant increase in the mean value of ESR and CRP in (active UC) in comparison with the other groups ${ }^{[2]}$

In another study, it was shown that FC was a highly useful biomarker than other blood parameters in the initial investigation of suspected pediatric IBD. FC level between 200 and $300 \mu \mathrm{g} / \mathrm{g}$ yielded an optimum sensitivity of $89-93 \%$ and specificity $74-83 \% .{ }^{[24]}$ In a meta-analysis, of 30 studies that included 5983 patients, FC was higher in IBD patients than in non-IBD patients (by $219 \mu \mathrm{g} / \mathrm{g}$ ) with excellent pooled sensitivity and specificity in distinguishing between these groups (95 and $91 \%$, respectively). ${ }^{25]}$

In another meta-analysis, the diagnostic accuracy of FC in the evaluation of patients with suspected IBD was evaluated in 30 studies including 1041 patients (670 adults, 371 children). Pooled sensitivity and specificity rates of calprotectin testing were 93 and $96 \%$, respectively with significantly low specificity in children and teenagers $(76 \%)$. This meta-analysis stated that using FC as a diagnostic test in suspected adult IBD could result in $67 \%$ reduction in patients requiring endoscopy, but might also result in a delayed diagnosis in $6 \%$ of patients because of false-negative test results. ${ }^{[26]}$

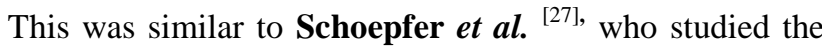
correlation between endoscopic activity on one hand, and FC, CRP, PLT, TLC, and the clinical score (Lichtiger Index) on the other hand. The best correlation was with FC, followed by the Lichtiger Index, CRP, TLC, and PLT. Fecal calprotectin (FC) was the only marker that could discriminate between different grades of endoscopic activity with a cut-off of $57 \mu \mathrm{g} / \mathrm{g}$, sensitivity $91 \%$, and specificity $90 \%$ to detect the active disease.

Also, Rodriguez-Moranta et al. ${ }^{[28]}$, found that FC correlated with the degree of inflammation better than other clinical indicators and serological markers. In addition, it could also be useful to predict mucosal cure and the risk of recurrence.

Similar results were reported in patients with IBD; endoscopic disease activity was highly correlated with FC $(\mathrm{r}=0.834)$, followed by the Clinical Activity Index $(\mathrm{r}=$ 0.672), CRP ( $r=0.503)$, and blood leukocytes $(r=0.461)$. The overall accuracy of calprotectin in detecting endoscopically active disease was $89 \%$ and it was the only marker to discriminate inactive, mild, moderate, and highly active disease. ${ }^{[20]}$

Lobaton et al. ${ }^{[29]}$, evaluated the ability of FC to predict endoscopic activity according to the Mayo score in 123 patients with UC. They found that FC was an accurate marker of endoscopic remission in UC.In general, FC values correlate better with endoscopic findings than with 
clinical status. So that, thay may detect residual inflammatory activity in patients with presumably quiescent disease. ${ }^{[30]}$

This is in agreement with Smith and Gaya, [31] who studied the utility of FC analysis in adult IBD and they found that FC correlated well with mucosal disease activity and this in turn made it useful for assessment of activity, monitoring response to treatment, and predicting relapse.

In conclusion: Fecal calprotectin (FC) is a valuable, simple, cost-effective, and noninvasive surrogate marker in evaluation of UC activity. It is better than CRP in discriminating mild cases from moderate to severe cases. Measurement of FC level can be recommended as a screening tool for patients with lower GI symptoms; also, serial measurements can be recommended for UC patients to assess disease severity, response to treatment, , and recurrence of activity. Further studies on a large number of patients are needed to determine the role of FC in the diagnosis of other organic colonic diseases in general and colonic malignancy in particular.

\section{REFERENCES}

1. Vermeire S G,Van Assche, Rutgeerts P. Laboratory markers in IBD: useful, magic, or unnecessary toys? Gut. 2006; 55 (3):426-31.

2. Mohamed A.E. Nouh, Atef A.E. Ali, Emad F.A. El Halim, et al. Calprotectin as a fecal marker for diagnosis and follow-up in patients with ulcerative colitis Menoufia Medical Journal 2014; 27 (1) :35-43.

3. Tibble JA, Teahon K, Thjodleifsson B, Roseth A, Sigthorsson G, Bridger S, et al. A simple method for assessing intestinal inflammation in Crohn's disease. Gut 2000; 47: 506-13.

4. Dolwani S, Metzner M, Wassell JJ, Yong A, Hawthorne AB. Diagnostic accuracy of faecal calprotectin estimation in prediction of abnormal small bowel radiology. Aliment PharmacolTher. 2004; 15; 20 (6): 615-21.

5. Tibble JA, Sigthorsson G, Bridger S, Fagerhol MK, Bjarnason I. Surrogate markers of intestinal inflammation are predictive of relapse in patients with inflammatory bowel disease. Gastroenterol 2000; 119 (1); 15-22.

6. Mindemark $M$ and Larsson A. Ruling out IBD: estimation of the possible economic effects of preendoscopic screening with F-calprotectin. Clin Biochem 2012; 45 (7-8): 552-55.

7. Laharie D, Mesli S, El Hajbi F, Wang JS. Prediction of Crohn's disease relapse with faecal calprotectin in infliximab responders: a prospective study. Aliment Pharmacol Ther 2011; 34 (4): 462-69.

8. Lasson A, Kilander A, Stotzer PO. Diagnostic yield of colonoscopy based on symptoms. Scand J Gastroenterol 2008; 43 (3): 356-62.

9. Tillett WS, Francis T. Serological reactions in pneumonia with a non-protein somatic fraction of pneumococcus. The Journal of Experimental Medicine.1930; 52 (4): 561-71.

10. Rudolph WG, Uthoff SM, McAuliffe TL, Goode ET, Petras RE, Galandiuk S. Indeterminate colitis: the real story. Diseases of the Colon and Rectum. 2002; 45 (11): 1528-34.
11. Abdelrazeq AS, Wilson TR, Leitch DL, Lund JN, Leveson SH. Ileitis in ulcerative colitis: is it a backwash?" Diseases of the Colon and Rectum. 2005; 48 (11): 2038-46.

12. Annaházi A, Molnár T, Farkas K, Rosztóczy A, Izbéki F, Gecse K, et al. Fecal MMP-9: a newnoninvasive differential diagnostic and activity marker inulcerative colitis. Inflamm Bowel Dis. 2013; 19 (2): 316-20.

13. D'Incà R, Dal Pont E, Di Leo V, Benazzato L, Martinato M, Lamboglia, et al. Can calprotectin predict relapse risk ininflammatory bowel disease? Am J Gastroenterol 2008; 103 (8): 2007-2014.

14. Langhorst J, Elsenbruch S, Koelzer J, Rueffer A, Michalsen A, Dobos G. Non invasive markers in the assessment of intestinalinflammation in inflammatory bowel diseases: performance of fecal lactoferrin, calprotectin, and PMN-elastase, CRP, and clinical indices. Am J Gastroenterol 2008; 103 (1):162-9.

15. Daperno M, Castiglione F, de Ridder L, Dotan I, Färkkilä M, Florholmen J, et al. Scientific Committee of the European Crohn's and Colitis Organization.Results of the 2nd part Scientific Workshop of the ECCO. II: Measures and markers of prediction to achieve, detect, and monitor intestinal healing in inflammatory bowel disease. J Crohns Colitis. 2011; 5 (5): 484-98.

16. Wagner M, Peterson CG, Ridefelt P, Sangfelt P, Carlson M. Fecal markers of inflammation used as surrogate markers for treatment outcome in relapsing inflammatory bowel disease. World J Gastroenterol. 2008; 14 (36): 5584-9.

17. Geboes K, Riddell R, Öst A, Jensfelt B, Persson T, Löfberg R. A reproducible grading scale for histological assessment of inflammation in ulcerative colitis. Gut 2000; 47 (4):404-9.

18. Xiang JY, Ouyang Q, Li GD, Xiao NP. Clinical value of fecal calprotectin in determining disease activity of ulcerative colitis. World J Gastroenterol. 2008; 14 (1): 53-7.

19. Canani RB1, de Horatio LT, Terrin G, Romano MT, Miele E, Staiano A, et al. Combined use of noninvasive tests is useful in the initial diagnostic approach to a child with suspected inflammatory bowel disease. J Pediatr Gastroenterol Nutr 2006; 42 (1): 9-15.

20. Schoepfer AM, Beglinger C, Straumann A, Trummler M, Vavricka SR, Bruegger LE, et al. Fecal calprotectin correlates more closely with the Simple Endoscopic Score for Crohn's disease (SES-CD) than CRP, blood leukocytes, and the CDAI. Am J Gastroenterol. 2010; 105 (1):162-9.

21. Schoepfer AM, Beglinger C, Straumann A, Trummler M, Renzulli P, Seibold F. Ulcerative colitis: Correlation of the Rachmilewitz endoscopic activity index with fecal calprotectin, clinical activity, C-reactive protein, and blood leukocytes. Inflamm Bowel Dis. 2009; 15 (12): 1851-8.

22. Røseth AG, Aadland E, Grzyb K. Normalization of faecalcal protectin: a predictor of mucosal healing in patients. Gastroenterology. 2004; 39 (10): 1017-20.

23. Mao R, Xiao YL, Gao X, Chen BL, He Y, Yang L, et al. Fecal calprotectin in predicting relapse of inflammatory bowel diseases: a meta-analysis of prospective studies. Inflammatory Bowel Diseases. 2012; 18 (10):1894-9.

24. Henderson P, Casey A, Lawrence SJ, Kennedy NA, Kingstone K, Rogers P, et al. The diagnostic accuracy of fecal calprotectin during the investigation of suspected pediatric inflammatory bowel disease. Am J Gastroenterol . 2012; 107 (6): 941-9. 
25. Von Roon AC, Karamountzos L, Purkayastha S, Reese GE, Darzi AW, Teare JP, et al. Diagnostic precision of fecal calprotectin for inflammatory bowel disease and colorectal malignancy. Am J Gastroenterol. 2007; 102 (4): 803-13.

26. Van Rheenen PF, Van de Vijver E, Fidler V. Faecal calprotectin for screening of patients with suspected inflammatory bowel disease: diagnostic meta-analysis. BMJ 2010; 341: c3369.

27. Schoepfer AM, Beglinger C, Straumann A, Safroneeva E, Romero Y, Armstrong D, et al. Fecal calprotectin more accurately reflects endoscopic activity of ulcerative colitis thanthe Lichtiger Index, C-reactive protein, platelets, hemoglobin, and blood leukocytes. Inflamm Bowel Dis. 2013; 19 (2): 332-341
28. Rodríguez-Moranta F, Lobatón T, Rodríguez-Alonso L, et al. Fecal calprotectin in the diagnosis of inflammatory bowel diseases. Inflamm Bowel Dis. 2013; 0:1-9, pii: S0210-5705 (13) 00004-6.

29. Lobatón T, Rodríguez-Moranta F, Lopez A, Sánchez E, Rodríguez-Alonso L, Guardiola J. A new rapid quantitative test for fecal calprotectin predicts endoscopic activity in ulcerative colitis. Inflamm Bowel Dis. 2013; 19 (5): 1034-42.

30. Sipponen T, Kolho KL. Faecal calprotectin in children with clinically quiescent inflammatory bowel disease. $S c$ and J Gastroenterol. 2010; 45 (7-8): 872-7.

31. Smith LA, Gaya DR. Utility of faecal calprotectin analysis in adult inflammatory bowel disease. World $J$ Gastroenterol. 2012; 18 (46): 6782-9. 